This is the author's copy of the publication as archived in the DLR electronic library at http://elib.dlr.de. Please consult the original publication for citation, see https://doi.org/10.2514/6.2021-3315.

\title{
Overall power train performance estimation from pseudo transient models
}

\section{Max Arzberger}

The generation of an overall propulsion system performance deck is key for the block fuel analysis of aircraft within an aircraft design workflow. In case of a hybrid electric propulsion system, it requires a sufficiently detailed modeling of the electric components and the turbomachinery to generate such a deck. The values for the performance deck then result out of the steady-state solution of the drive train that needs to be found robustly despite non-linearities and dealing with novel limitations resulting from the electric machines. This paper describes how the author met this challenge by building a framework for the hybrid electric deck generation on top of a dedicated object-oriented modeling library for hybrid electric propulsion. A hybrid-electric single-aisle aircraft with an aft fan for boundary-layer ingestions thereby represents the prime application example.

\section{Copyright Notice}

Copyright $\odot 2021$ by German Aerospace Center (DLR). Published by the American Institute of Aeronautics and Astronautics, Inc., with permission.

Arzberger, Max (2021) Overall power train performance estimation from pseudo transient models In: AIAA Propulsion and Energy 2021 Forum, 2021. AIAA/IEEE Electric Aircraft Technologies Symposium (EATS), 11-13 Aug 2021, virtual event. DOI: 10.2514/6.20213315 


\title{
Overall power train performance estimation from pseudo transient models
}

\author{
Max J. Arzberger ${ }^{1}$ \\ German Aerospace Center, Oberpfaffenhofen, 82234, Germany
}

\begin{abstract}
The generation of an overall propulsion system performance deck is key for the block fuel analysis of aircraft within an aircraft design workflow. In case of a hybrid electric propulsion system, it requires a sufficiently detailed modeling of the electric components and the turbo-machinery to generate such a deck. The values for the performance deck then result out of the steady-state solution of the drive train that needs to be found robustly despite non-linearities and dealing with novel limitations resulting from the electric machines. This paper describes how the author met this challenge by building a framework for the hybrid electric deck generation on top of a dedicated object-oriented modeling library for hybrid electric propulsion. A hybrid-electric single-aisle aircraft with an aft fan for boundary-layer ingestions thereby represents the prime application example.
\end{abstract}

\section{Nomenclature}

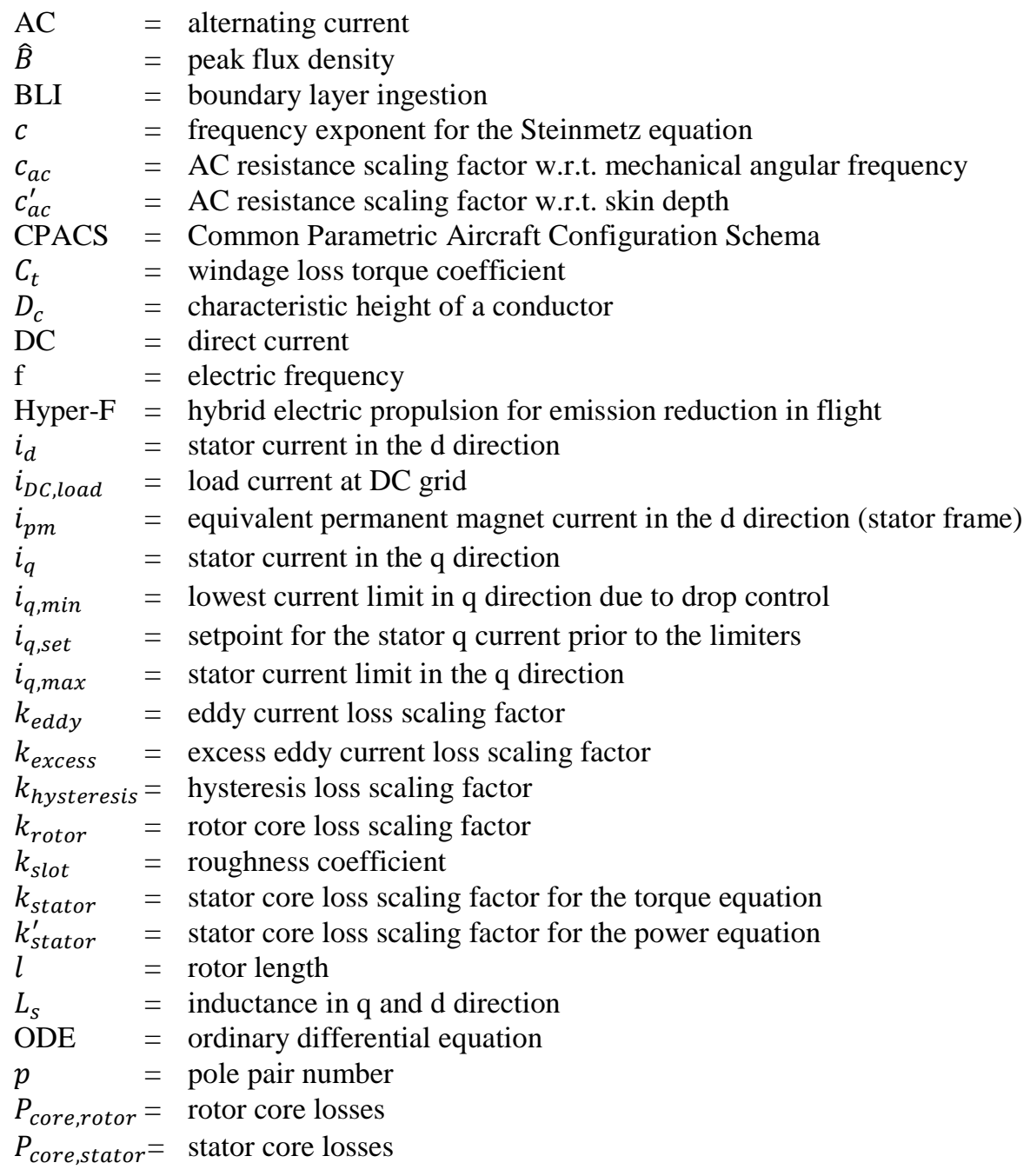

\footnotetext{
${ }^{1}$ Research engineer, Institute of System Dynamics and Control.
} 


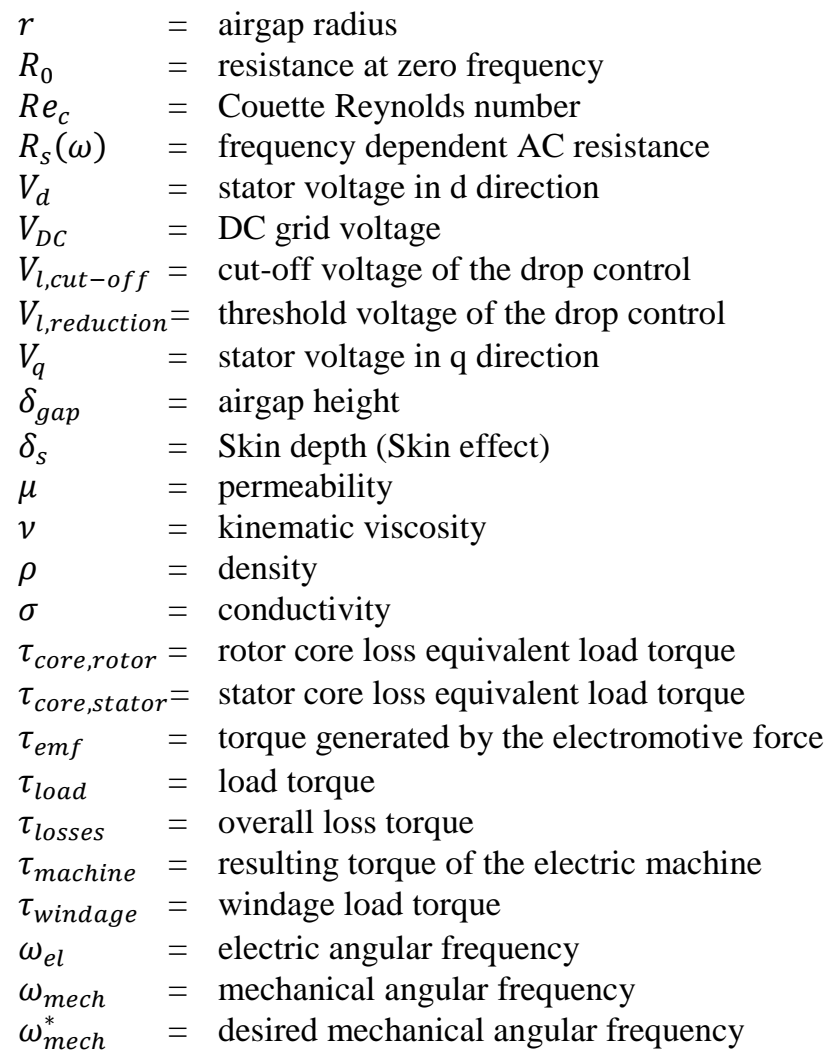

\section{Introduction}

Hybrid-electric propulsion systems are increasingly seen as game changer for future aircraft. As pointed out in Ref. [1], there are still great uncertainties regarding the trades in the design space of hybrid electric aircraft. In literature, a wide variety of approaches varying in their level of fidelity can be found.

Many design studies are addressing hybrid electric aircraft trades from the aircraft design perspective. Typically, the additional power train components are modeled with fixed key performance indicators such as power specific weight estimates and constant efficiencies [2,3,4]. This high level allows for a quick evaluation of different configurations but comes at the cost of omitting more detailed considerations like electric machine topology and direct component interaction. Supplementary, analysis from the propulsion system perspective has been performed (e.g. [5]).

It is uncertain how the results of these high-level and discipline-based studies hold up to scrutiny when the design is detailed. To address this query, studies have been conducted based on multidisciplinary design environments coupling automatized higher order component sizing tools to assess the benefit on aircraft level [6] and propulsion system level [1]. These approaches account for the coupling and snowball effects implicitly and allow for projection of the detailed components sensitivities onto aircraft level. To limit the design space, a fixed set of component topologies and predefined operation strategies is considered.

This paper provides a key enabler for a similar close coupling of detailed propulsion system design tools with the overall aircraft design. Therefore, the author introduces a framework which allows to derive a performance model using standard turbomachinery decks as defined in the CPACS standard [7] and detailed electric machine models to obtain the steady state performance of a hybrid electric powertrain that can be directly inserted into the overall aircraft design methodology used in pre-studies. In particular, following challenges are addressed:

- The desired framework has to obey the interface to overall aircraft design workflow and yield the result in an expected format. Furthermore, the component models have to be adapted to the parameters and performance description provided by the underlying component design methodologies.

- The propulsion system modeling approach of the framework needs to be modular so that a large variety of possible hybrid-electric power trains can be modeled with moderate effort.

- The modeling of electric machines deserves special attention, in order to include the results of an available detailed design into the established design processes.

- The resulting equation-system for the steady-state solution of the power train is non-trivial to solve. Hence, a robust solution method is needed for the generation the hybrid electric propulsion system deck. Therefore, the author has chosen to use prototypical control dynamics so that an ODE-solver can reliably approach the convergence area of the steady-state solution.

The following sections address these challenges one-by-one, starting with the Hybrid electric Propulsion for Emission Reduction in Flight (Hyper-F) project. 


\section{Integrating the performance estimation into the Hyper-F project workflow}

The European Union is eager to decrease the emissions in the aviation sector by developing novel aircraft as well as propulsion technologies and defined related key targets in flight path 2050 [8]. In the European project Hyper-F, a STARC-ABL like aircraft (Fig. 1) with turboelectric propulsion system architecture (Fig. 2) and boundary layer ingestion (BLI) is considered. The architecture harnesses the momentum deficit created by the fuselage with an electrically powered aft fan: the BLI fan. The electric power is provided via generators driven by turbofan engines. Hence, there are no batteries involved. The potential of fuselage boundary layer ingestion has been investigated in Ref. [9]. In previous research, studies have been conducted [3, 2] from the aircraft design perspective. In both cases, power specific weight estimates for the components were used as lumped parameters for the sizing of the power train. Thus, the detailed sizing of the electric components, implications resulting from torque/ current limits of the electric machines, noise, fan boundary layer interaction as well as structural implications on the fan due to the BLI were not considered. In a next step of the project Hyper-F fills this gap. In the following sections, the detailing of the power train performance evaluation is addressed.

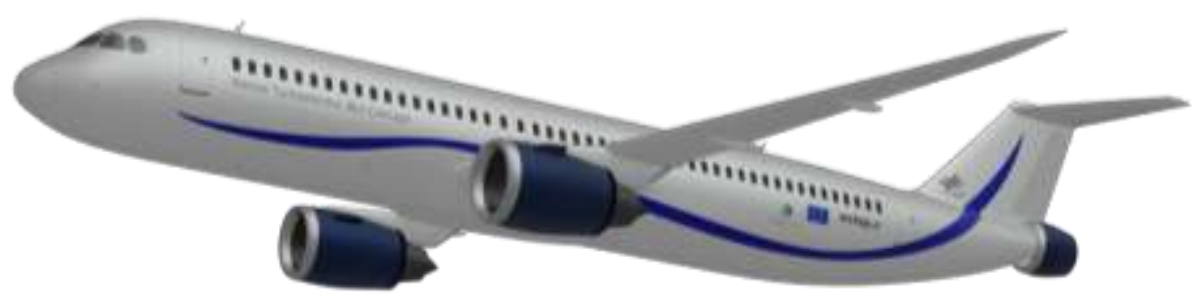

Fig. 1: Partial turboelectric BLI concept with in Hyper-F [3]

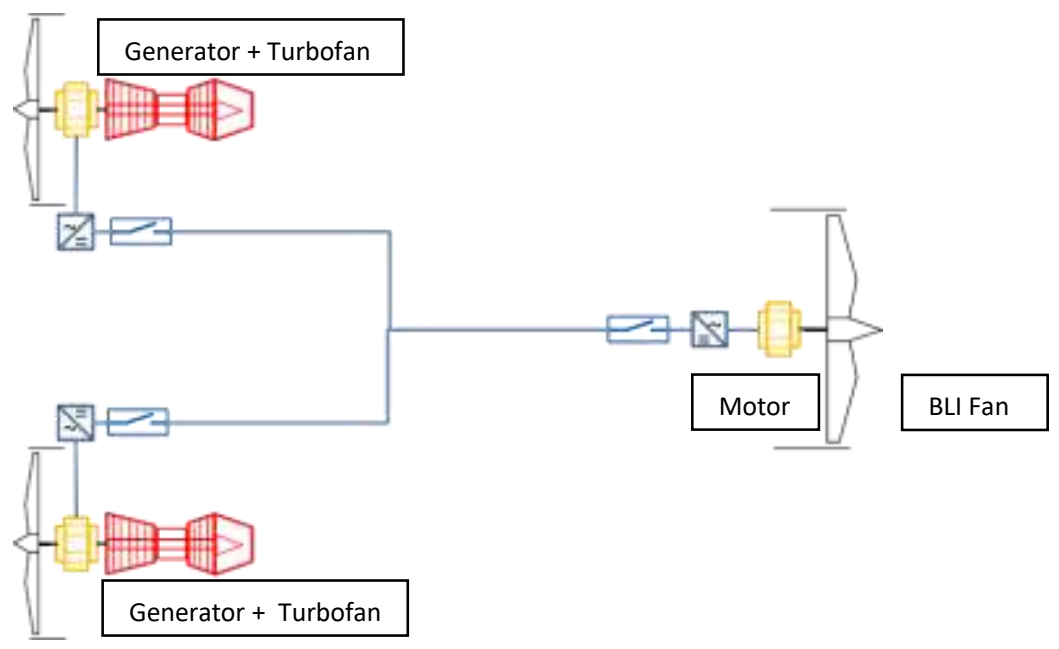

Fig. 2: Simplified architecture schema for the power train

The performance assessment of the hybrid electric aircraft concept is based on the evaluation workflow of the project depicted in Fig. 3. As the BLI fan, the turbofan and the electric machine design is conducted at a high-fidelity level, the different tasks are performed sequentially and the interfacing of the tools is done manually. As high-fidelity component design and analysis is time consuming, a specific design point needs to be pre-selected prior to the detailed analysis. This preselection is based on previous work, namely the design space exploration on L0 level published in Ref. [3]. The identified optimal thrust split for mid cruise serves as starting point for the detailed analysis.

As input to the aerodynamic fan design, the boundary layer profile is estimated. The fan design point is based on the targeted mid cruise operation determined in design space exploration. The detailed fan design yields characteristics for the fan cycle calculus which generates the respective BLI fan performance deck.

For the propulsions system design, a take-off, an end of field, a top of climb and a mid-cruise (design point) requirement are set. Breaking these top-level requirements into component requirements calls for an operation strategy determining thrust split in the off-design and therefore the related BLI fan operation. From the BLI fan operation, the motor requirements in means of a torque corner point can be determined and the requirements for the electric component chain can be deduced. For the generator design, a preliminary assumption for the shaft speeds of the gas turbine is to be taken. By following the efficiency chain of the electric transmission system, the 
shaft power off-take at the turbofan engines is computed for all considered requirements. Together with the BLI thrust share, the complete turbofan requirements in means of remaining thrust requirement and shaft power offtake become available. Based on the resultant turbofan design, an turbofan engine performance deck is generated via cycle calculus.

Finally, the results from the high-fidelity component sizing are to be projected on the aircraft level to allow for an adaption of the LO aircraft design and to estimate the overall benefit compared to a baseline design in means of block fuel. Hence, a surrogate model of the overall propulsion system performance is identified as missing link and the related framework is addressed throughout this publication. To interface with the mission analysis in the overall aircraft design, the quasi steady state performance of the power train is to be delivered in the standard CPACS engine deck definition [7].

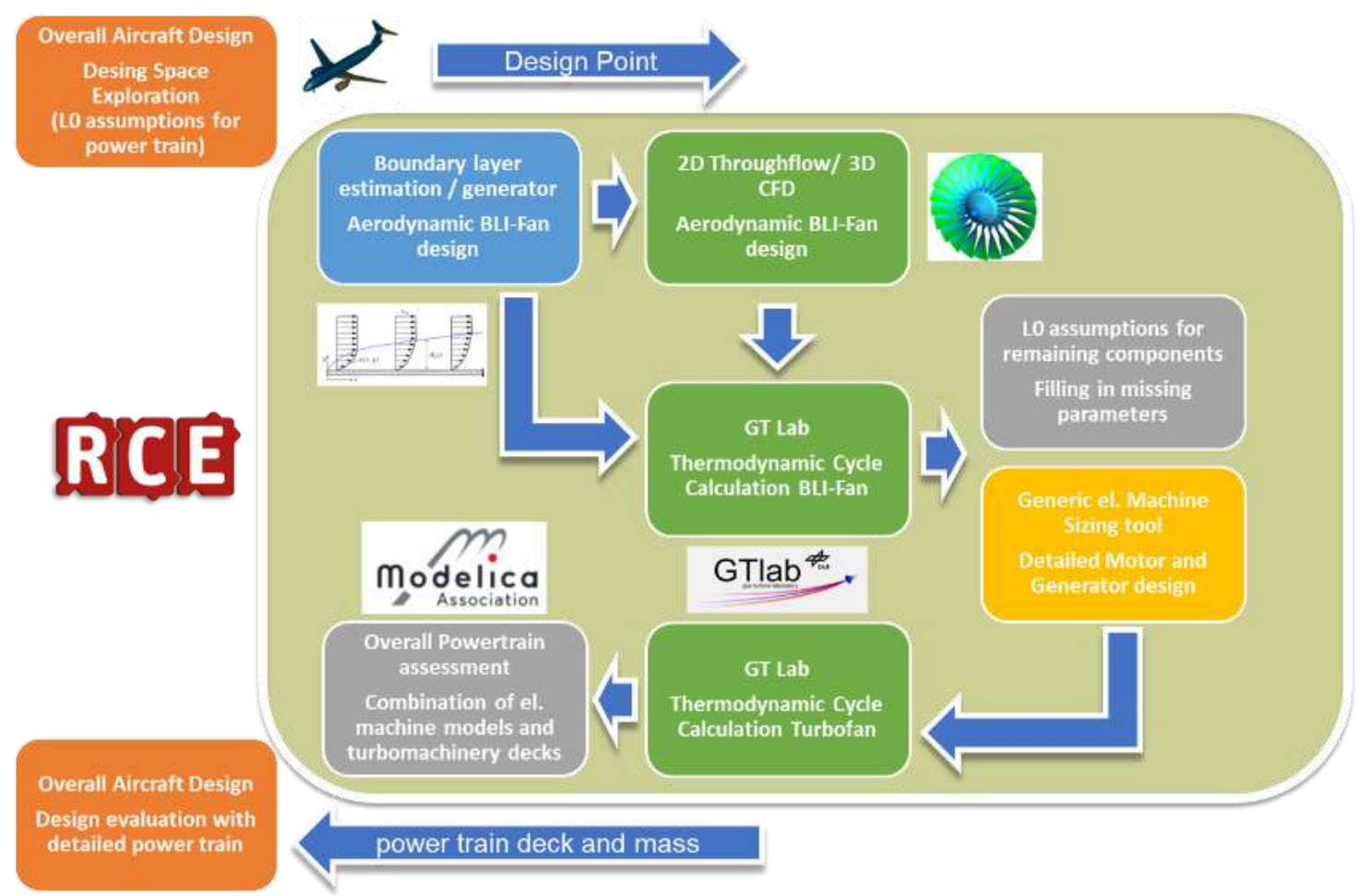

Fig. 3: Workflow of the Hyper-F project containing the powertrain performance evaluation framework described in this paper (grey box in the bottom left).

\section{Framework overview}

A dedicated library for the modeling of hybrid-electric powertrains has been created using the object-oriented equation-based language Modelica [10]. The central element of the framework is a propulsion system model based on this library (center of Fig. 4). The performance deck for the propulsion system is then obtained by running multiple simulations of the Modelica propulsion system model to obtain steady-state operation points of the propulsion system. These simulation runs are automated via Python. In a final step, the generated data is postprocessed and converted to the standard propulsion system deck structure defined by the CPACS standard (compare Ref. [7]). Prior to the simulation runs, the input parameters delivered by the respective component design tools are preprocessed and integrated into the component models. 


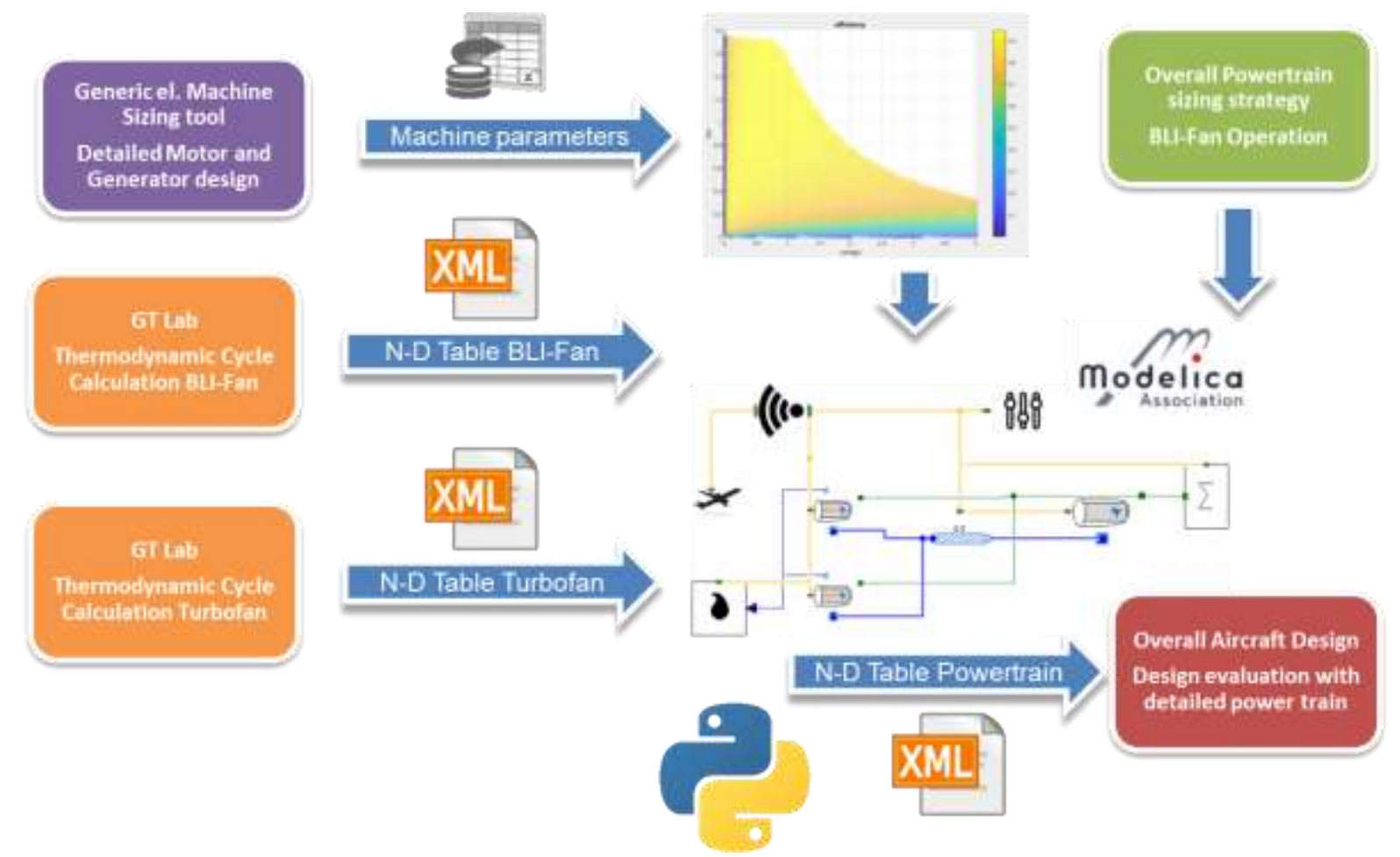

Fig. 4: Framework overview depicting the input data (arrows upper left position), the overall power train model for the STARC-ABL like architecture (central position) and the output generated with the automated simulation runs (bottom right position)

\section{Modeling the Electric Machines}

Compared to the overview paper on the library in Ref. [10], the model of the electric machines is extended to capture a greater level of detail. The modeling was coordinated with an industrial project partner such that parameters of the tool used for sizing the electrical machines can be directly applied to the electric machine model.

Even though a variety of potential motor/ generator topologies were considered in the project, the efforts are focusing on the most promising candidate: surface mounted permanent magnet synchronous motors. The electric machine models constitute a fundamental frequency model in the $\mathrm{d}-\mathrm{q}$ frame which is common for in the field of motor control. ${ }^{2}$ This level of detail reveals that the desired torque/ active power can be realized for a multitude of power factors. However, it does only cover resistive losses and by definition neglects harmonics. The subsequent paragraphs cover how the considered loss mechanism are integrated into this model. Since the output parameters of the preliminary sizing tool are limited to the design point losses, geometric dimensions and equivalent circuit parameters, standard loss correlations are used to identify power laws for extrapolating these losses based on the provided parameters. Hence, the approach has similarities with Ref. [11].

As pointed out in Ref. [12], the AC copper losses cannot be neglected for a wide span of electric machine designs. The frequency dependent AC copper losses result from the skin and proximity effect and affect the conductive area available for the electrons. Commonly, the analysis of AC copper losses is performed via computationally expensive finite element analysis and is highly dependent of the electromagnetic machine design $[13,12]$.

As measure for the impact of the effect, the skin depth (Eq. (1)) is compared with the characteristic height $D_{c}$ of the conductor.

$$
\delta_{s}=\sqrt{\frac{2}{\sigma \omega_{e l} \mu}}
$$

Therein, $\omega_{e l}, \sigma$ and $\mu$ are the electric angular frequency, the conductivity and permeability of the conductor material, respectively. The ratio $\frac{D_{c}}{4 \delta_{s}}$ relates to the total impact of the skin effect. Hence, the maximal ratio can be

\footnotetext{
${ }^{2}$ For further details the reader is referred to standard literature like Ref. [29] .
} 
utilized to select a proper model for the skin effect. In the model, the electric frequency is inferred by the shaft speed $\omega_{\text {mech }}$ and the pole pair number $p$ :

$$
\omega_{e l}=\omega_{m e c h} * p
$$

The selected AC resistance model follows a simple polynomial correlation which is fit to higher fidelity data obtained from the motor sizing tool:

$$
R_{s}\left(\omega_{m e c h}\right)=R_{0} *\left(1+\frac{c_{a c}^{\prime}}{\delta_{s}^{4}}\right)=R_{0} *\left(1+c_{a c} \omega_{m e c h}^{2}\right)
$$

Therein, $R_{0}$ represents the resistance value at zero frequency and $c_{a c}^{\prime}$ as well as $c_{a c}$ are scaling factors for the polynomial equation. The model captures the frequency dependency from the basic equations on circular and rectangular conductors described in Ref. $[14,15]$ and is valid if the characteristic height of the conductor is small compared to the minimal skin depth $\left(\frac{D_{c}}{4 \delta_{s}}<1\right)$ [16]. For this polynomial fit, the computational effort on the sizing tool side is limited to the computation of the resistance at zero frequency $R_{0}$ and the nominal operation point. The accuracy is yet limited since the impact of harmonics as well as the proximity effect are lumped into the $c_{a c}$ coefficient. This is especially true for higher frequencies, where the impact of $2 \mathrm{D}$ effects becomes more significant [13] and the most significant harmonics escape the range of validity of Eq. (3) [15].

The core loss models are separated for the rotor and the stator. As the rotor turns at the fundamental frequency it perceives only time constant stator quantities in a fundamental frequency model. Especially for electric machines controlled by converters, harmonics of the fundamental wave are present in the stator currents. These induce varying fields in the rotor causing hysteresis in the flux carrying rotor iron and eddy current losses in the iron as well as the magnets. Also, a sinusoidal current distribution in the airgap can only be approximated even for machines with distributed windings. Combined with the winding grooves, which yield small irregularities in the magnetic layout of the machine, they create spatial harmonics which contribute to the afore mentioned core losses. All these effects are assumed to scale with the fundamental electric frequency and hence the shaft speed. To include the core losses, Steinmetz equations are used. For further background information on Steinmetz based hysteresis modeling, the reader is referred to Refs. $[17,18]$. According to the design, the eddy current losses in the magnets dominate in the rotor. Hence, the classic split of the core losses into hysteresis, eddy current and excess eddy current losses (compare e.g. Ref. [17]) is assumed to be dominated by the eddy current loss term:

$$
\begin{array}{r}
P_{\text {core }, \text { rotor }}=k_{\text {hysteresis }} * f * \widehat{B}^{2}+k_{\text {eddy }} * f^{2} \widehat{B}^{2} \\
+k_{\text {excess }} * f^{1.5} \widehat{B}^{1.5} \approx k_{\text {eddy }} * f^{2} * \hat{B}^{2}
\end{array}
$$

Therein, $\widehat{B}^{2}$ and $f$ represent the peak magnetic flux density and the electric frequency, respectively. $k_{\text {hysteresis }}$, $k_{\text {eddy }}$ and $k_{\text {excess }}$ are scaling coefficients which depend on the design and the selected materials. Again, this loss term is used to generate a power law, based on the variables of the equivalent circuit model. To include this loss in the model, it is expressed in means of a load torque dependent on the shaft speed and the stator currents $\left(i_{d}, i_{q}\right)$ and a scaling factor $k_{\text {rotor }}$ :

$$
\tau_{\text {core }, \text { rotor }}=k_{\text {rotor }} * \omega_{\text {mech }} *\left(i_{q}^{2}+i_{d}^{2}\right)
$$

In the stator, varying stator currents as well as the magnetic field of the rotor induce stator hysteresis and eddy current losses. Besides the fundamental frequency content, also the harmonics in the stator currents contribute to these losses. Similar to the rotor, sensitivity to the shaft speed is assumed and only one natural Steinmetz term is considered:

$$
P_{\text {core }, \text { stator }} \approx k_{\text {stator }}^{\prime} * f^{c} * \widehat{B}^{2}
$$

Therein, $k_{\text {stator }}^{\prime}$ is a scaling factor and $c$ represents the frequency exponent for the Steinmetz equation. An applicable range of values for $c$ is given by Refs. $[18,19]$. This loss correlation yields the equivaled load torque in terms of a scaling factor $k_{\text {stator }}$, the stator $i_{q}$ current, the equivalent permanent magnet current $i_{p m}$ and the shaft speed $\omega_{\text {mech }}$ :

$$
\tau_{\text {core }, \text { stator }}=k_{\text {stator }} * \omega_{\text {mech }}^{c-1} *\left(i_{q}^{2}+i_{p m}^{2}\right)
$$


The formulation of the rotor and the stator core losses, reveal that saturation in the flux carrying material and the impact of the $i_{d}$ current on the stator losses are neglected. The author further neglects the direct impact of the core losses on the electric properties by modeling them as load torques. Alternative to the selected approach, the core losses can also be included by inserting an additional resistor into the classical d, q model (e.g. Refs. [20, 21]).

While friction in the bearings is omitted, windage losses in the airgap are accounted for. These are based on Ref. [22]. Therein, a roughness coefficient $k_{\text {slot }}$ is used to adapt the windage loss estimation of smooth cylinders for machines with rotor and/or stator slots:

$$
\tau_{\text {windage }}=k_{\text {slot }} * C_{t} * \pi \rho \omega_{\text {mech }}^{2} r^{4} l
$$

with airgap radius $r$, rotor length $l$, air density $\rho$ and windage loss torque coefficient $C_{t}$.

The windage loss torque coefficient $C_{t}$ depends on the flow regime which can be characterized via the Couette Reynolds number:

$$
R e_{c}=\frac{\omega_{m e c h} * r * \delta_{g a p}}{v}
$$

where $\delta_{g a p}$ and $v$ are the airgap height and the kinematic viscosity of air, respectively. As in the prefiguration, the regime-wise torque coefficient definitions published in Ref. [23] are used. The impact of the flight level on the windage losses is neglected due to integration uncertainties.

As previously indicated, saturation is neglected and the $\mathrm{d}$ and $\mathrm{q}$ inductances are considered to have a constant value of $L_{s}$, although permanent magnet synchronous machines commonly operated close to the saturation of the magnetic material in the design point [19]. Hence, close to the design point the inductances in the equivalent circuit model usually enter a nonlinear region [19]. Furthermore, the flux in both directions cannot be superposed anymore and a high current magnitude in one direction influences the induction in the other direction ("cross-saturation") $[19,24]$.

Concluding, the final set of equations for the stator voltages $\left(V_{d}, V_{q}\right)$ and the resultant machine torque $\left(\tau_{\text {machine }}\right)$ is:

$$
\begin{gathered}
V_{d}=R_{s}\left(\omega_{\text {mech }}\right) * i_{d}-L_{s} p \omega_{\text {mech }} i_{q} \\
V_{q}=R_{s}\left(\omega_{\text {mech }}\right) * i_{q}+L_{s} p \omega_{\text {mech }}\left(i_{d}+i_{\text {pm }}\right) \\
\tau_{\text {machine }}=\underbrace{1.5 * p L_{s} i_{\text {pm }} * i_{q}}_{-\tau_{\text {losses }}} \underbrace{-\tau_{\text {winerestator }}-\tau_{\text {coreror }}-\tau_{\text {core }}}_{\text {windage }}
\end{gathered}
$$

As indicated by Eq. (10) - (12), the electric time constant resulting from the inductances are neglected as well.

The equivalent circuit model parameters are inputs directly delivered by an industrial partner. For the different loss mechanisms, the design point losses are delivered as well. The design point losses are matched via scaling factors present in the deduced formulas, namely $c_{a c}$ for the AC copper losses, $k_{\text {rotor }}$ for the eddy current losses in the rotor, and $k_{\text {stator }}$ for the core losses in the stator. The windage loss estimation, however, only relies on the geometric parameters delivered.

\section{Powertrain Control Scheme and Steady-State Evaluation}

To retrieve the values for the performance deck, only data on steady-state operation is needed. However, the direct evaluation of the steady-state system is practically often unfavorable since the resulting non-linear equation system is difficult to solve robustly. This is especially true for the wide range of considered operation points since the overall system has to be compliant with all component limits. E.g. in the exemplary application case, different thrust level at grid of 9 different Mach numbers and 41 flight levels were considered (Fig. 5) yielding a total of about 4000 operating points. Also, there might be multiple spurious non-desired steady-state solutions that shall be avoided. 


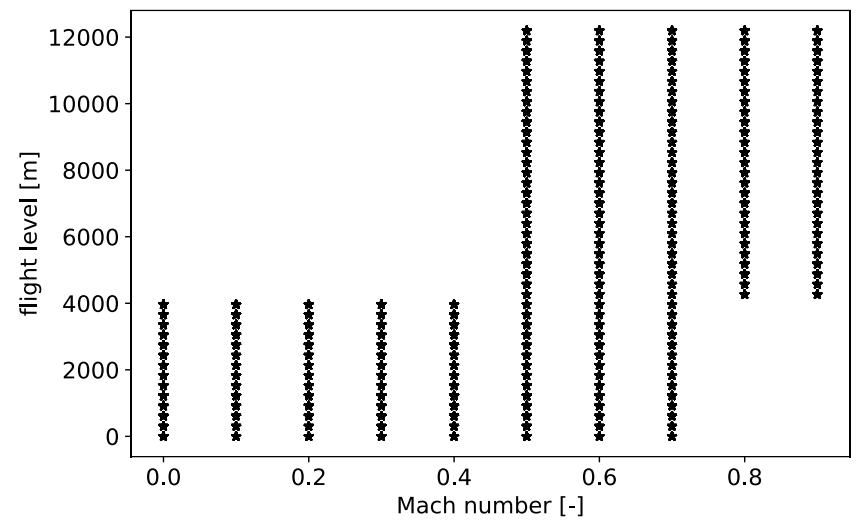

Fig. 5: Exemplary raster in the dimensions Mach number and flight level for a deck generation

Since the underlying library supports the modeling of transient behavior, it is a natural choice to use an ODEsolver to approach the desired steady state. Due to the transient modeling, also control tasks have to be addressed, such that a coordinated operation of the components is granted. This might seem to be a disadvantage at first. Yet, implications resulting from control and management become visible. Also, the implementation of the load coordination via component controllers gives an intuitive implementation of the management. In the following, the author describes the applied control scheme for the hybrid-electric powertrain. Therefore, this section guides the reader from the electric load(s) to the electric sources in order to describe the used prototypical approach of management and control.

The framework is solely intended to generate an overall steady state performance description based on an operation strategy and parametrized component models. Hence, the focus is on obtaining a steady state operation point for any set of input parameters. Thus, we can reduce the effort on envelope protection in the control and management by handling remaining ill-posed input parameter sets in post-processing.

This also eases to comply with one of the major focuses of the framework: the modularity which is achieved best if the underlying approaches allow for a plug and play like adaption of the architecture. As distributed microgrid control features similar problems statements [25], the used mechanisms serve as blue print for the proposed approach.

Since the inlet Mach number and the environment conditions depend on the flight condition, the only means of changing the thrust of a fan is adapting the shaft speed (assuming fixed nozzle and pitch). By modeling a rotational inertia for the shaft of an electrically driven fan like the BLI fan, the shaft speed becomes a state variable. Dependent on the flight state and the shaft speed state, the complete fan operation in terms of thrust and load torque $\tau_{\text {load }}$ is determined.

The shaft speed state can be governed by the motor torque as control variable to obtain a desired value $\omega_{\text {mech }}^{*}$. Typically, the shaft speed control loop of the motor control determines the currents related to the desired torque. These currents have to comply with the motor limits (voltage and current) and define the current setpoints for the inner current control loops of a classic cascaded control.

For the outer loop, a maximum torque per ampere control is selected for the base speed range of the electric machine. Since a surface mounted permanent magnet synchronous motor is assumed, the motor does not generate any reluctant torque. Thus, this control only varies the $i_{q}$ current up to the nominal speed, where the voltage limit comes into play. Beyond the base speed range, a maximum torque per ampere approach is followed up to the voltage limit. After hitting the voltage limit, the $i_{d}$ current is utilized to counteract the rotor field such that the voltage limit is complied with. As the vector sum of the currents in $\mathrm{d}$ and q direction is subject to the current limit, the $i_{d}$ current affects maximum current available for torque generation $i_{q, \text { max }}$. Thus, by surpassing the base speed range, the motor performance switches from a torque limited to a power limited operation.

As the controls only govern a virtual system, some short cuts can be taken: modeling the power electronics as ideal components and omitting the electric time constant of the electric machine allow for directly setting the currents in $\mathrm{d}$ and $\mathrm{q}$ direction. Hence, the inner current control loops are hidden in the modeling of the converters and electric machines. So, the classic cascaded electric machine control condenses to a limited PI controller for the outer loop prescribing the $i_{q}$ current demand/ the related torque (Fig. 6). Utilizing that all parameters of the electric machines are known a priori and assuming a constant distribution grid voltage $V_{d c}$, a lookup table for the maximum torque generating current $i_{q, \max }$ over the mechanical shaft speed is precomputed. By limiting the output 
of the PI controller, it is granted that the $i_{d}$ required to comply with the voltage limit of the machine does not violate the current limit. For the generator mode, an analogue approach is followed to obtain a maximum negative $i_{q}$ current look up table. As the PI controller output is limited, an anti-windup is required. The desired value for $i_{d}$ is subsequently generated directly from a precomputed lookup table based on the shaft speed and the $i_{q}$ current demand. Similar to $i_{q}, i_{d}$ is directly enforced on the machine via the ideal power electronics model.

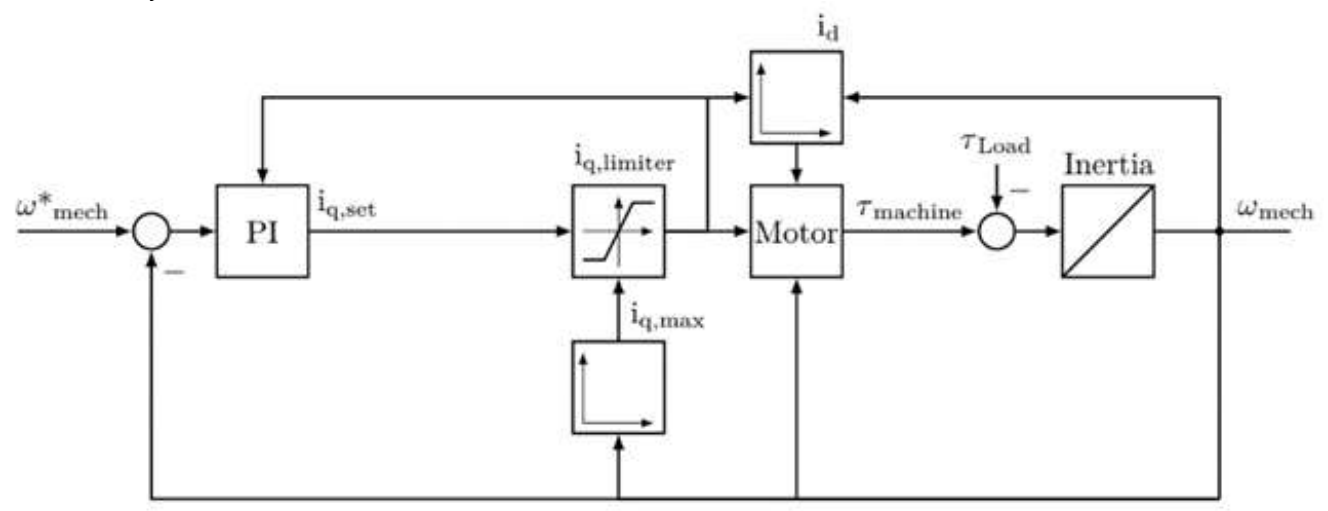

Fig. 6: Block diagram of the basic motor control

In micro grids, commonly the DC capacitance of the power electronics or a dedicated capacitor bank are harnessed to obtain the grid state (e.g. Refs. [25, 26]). Following this approach and modeling a DC grid capacitance, power balancing can be enforced by controlling the DC grid voltage with the generators. The control follows analogous to the motor but the PI controller is used to govern the voltage of the grid/ DC link capacitor $V_{D C}$ by setting a negative $i_{q}$ current instead of controlling the shaft speed of the fan with a positive $i_{q}$ current. The power balancing of electrically driven fans like the BLI fan which are modeled in the $\mathrm{d}$, $\mathrm{q}$ frame and the DC grid is based on a real power balance and determines the current on the DC side $i_{D C, \text { load }}$ :

$$
V_{D C} * i_{D C, \text { load }}=\frac{3}{2}\left(i_{q} * V_{q}+i_{d} * V_{d}\right)
$$

In addition, an assumed constant efficiency of the converter is taken into account. Since, the shaft speed control and the motor state currents fully determine the right-hand side of the power balance, electrically driven fans appear as a constant power load on the DC side.

For the base speed range, the power capability of generator is dependent on its shaft speed. Hence, for operating points with low turbofan power, the generators operate at a comparable low shaft speed and potentially cannot fully power the BLI motor. If the related top-level power management (secondary level) does not adjusts the BLI setpoint accordingly, a fall back protection level (primary level) of the modeling library described in Ref. [10] takes effect. In this fallback level adapted from common DC-grid power management, the loads monitor the grid voltage. Given that the sources are not capable of maintaining the DC-grid voltage within a certain voltage band around the nominal voltage $V_{D C}^{*}$, the loads power down to prevent a fallout. To do so, the maximum allowable power of the loads is linearly reduced in a second voltage band defined by $V_{l, \text { reduction }}$ and $V_{l, \text { cut-off }}$ (Fig. 7).

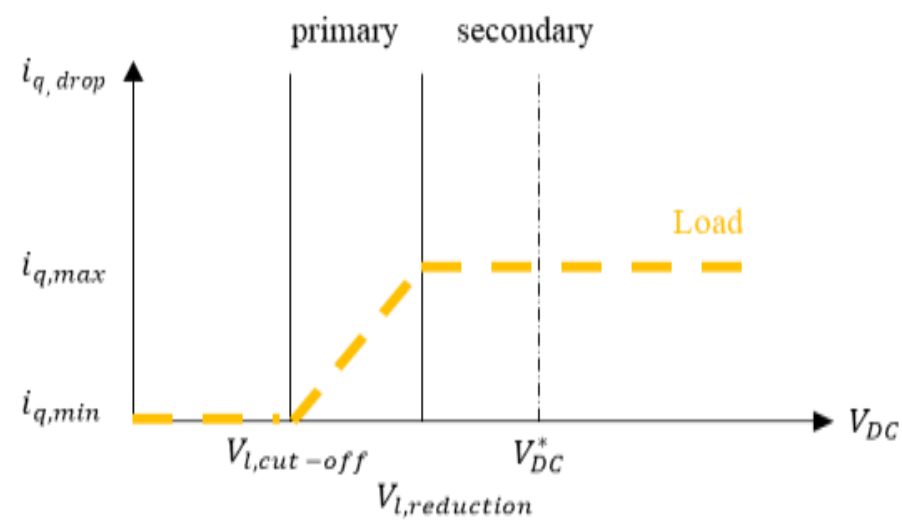

Fig. 7: Drop characteristic for the load shedding 
This reduction is implemented by a second, grid voltage dependent limiter applied to the $i_{q}$ current (Fig. 8) linearly reducing the limit of the $i_{q}$ current to $i_{q, \min }$ and can be interpreted as a drop control of the grid voltage $V_{D C}$ performed on the load side. Hence, grid stability issues resulting from constant power loads (as mentioned in e.g. Ref. [27]) can be circumvented and the grid voltage can only drop to the cut-off voltage $V_{l, c u t-o f f}$. Thus, the new limiter performs a load shedding by overruling the load controller after surpassing a threshold voltage $V_{l, \text { reduction. }}$. Hence, the onset of this new limiter at the threshold grid voltage can be interpreted as a mode switch from a task-oriented load control to a grid stabilizing load shedding drop control.

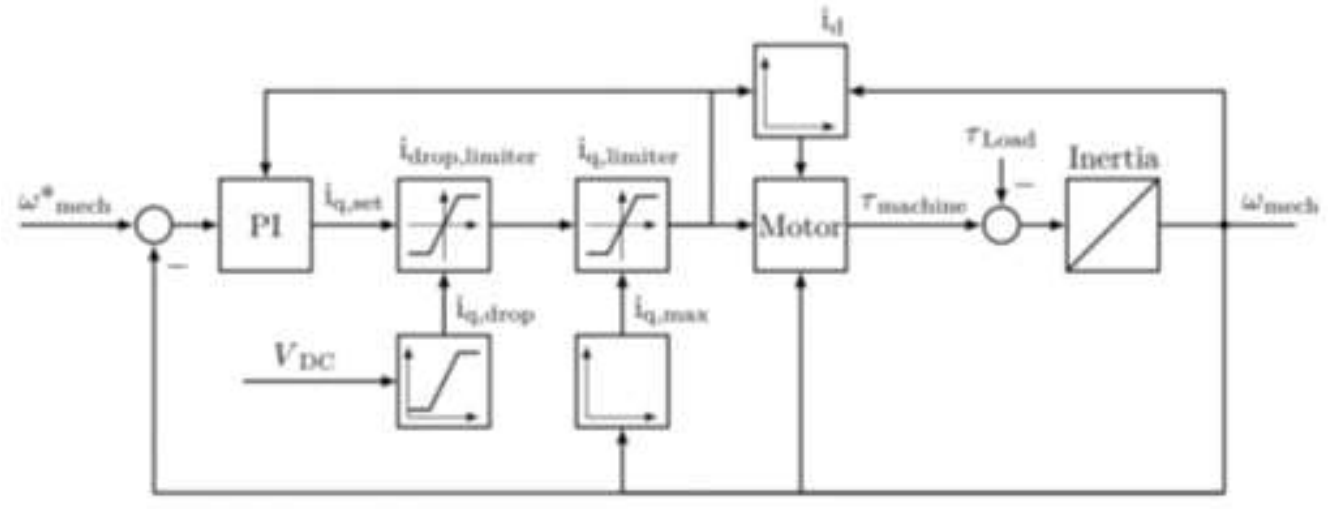

Fig. 8: Block diagram of the motor control including load shedding control

Given that the load shedding limiter potentially reduces the allowable $i_{q, \max }$ to zero $\left(i_{q, \min }=0\right)$ after meeting $V_{l, c u t-o f f}$, this limiter potentially breaks the feed back for the subsystem control in Fig. 8. In case the open loop system is stable, it can still converge to a steady state operation point. For an electrically driven fan and an open control loop, the steady state operation point would coincide with wind milling operation of the fan. Yet, the fan deck generated from the cycle calculus only reaches points with a remaining surge margin and omits the wind milling operation range. This problem can be met by either limiting down to a remaining $i_{q}$ current allowing a fan operation within the target surge margin, by proper extrapolation of the fan deck, or by actually capturing the wind milling performance deck. For a physical system and actual transient system models, the first approach is the proper choice. Since this approach complexifies the drop characteristic, the second approach can be applied for the deck generation. However, operation points where the fan deck is extrapolated need to be removed in postprocessing to prevent errors in the overall performance estimation.

Typically, the Full Authority Digital Engine Control (FADEC) powers the turbofan according to a thrust lever position set by a pilot. Moreover, it performs an envelope protection to prevent surge in the compressor stages and the fan, lean blow out, exceeding of the maximum turbine inlet temperature... For the problem at hand, a more abstract approach can be followed, as we are only interested in converging to numerous steady state operating points covering the whole operation range. Furthermore, the turbofan deck only contains operating points inside the envelope and the envelope narrows down the allowable operation range for an increase of power offtake. Besides the internal degrees of freedom (e.g. cooling air flow, variable nozzles, variable bleed valve) hidden in the performance deck, the remaining control variable is the fuel flow fed to the engine.

The performance deck is based on quasi steady state operation points described by a set of flight level, Mach number, the low-pressure shaft speed, fuel air ratio/fuel mass flow and low-pressure shaft excess torque. It only contains stable operating points. The excess torque is intended to counteract the generator torque and is therefore related to the generator power offtake in quasi steady state operation. Similar to an electric fan, the low-pressure shaft speed can be turned into a state by implementing a low-pressure shaft inertia. Again, the turbomachinery torque and the generator torque act on the inertia as source and load torque, respectively. Since both turbofans and the attached generators are operated symmetrically, the related control and input signals can be provided by one shared source.

As the previous sections argue for the distribution network being stable even in absence of any electric source due to the load shedding drop control, we can employ the same drop control approach to stop the generators from decelerating the turboshaft beyond a pre-defined low-pressure shaft speed. This drop control is then based on the low-pressure shaft speed instead of the grid voltage. Furthermore, we can also allow for extrapolation of the performance deck and remove infeasible operation points in post-processing.

For the deck generation, the operation range of the power train is rasterized in means of flight Mach number, flight level and fuel air ratio by directly setting them as input parameters for the turbofans. The reminder of the power train, including the low-pressure shaft speed, adjusts its setpoints according to the available power and the 
electric machine limits at the basis of the afore mentioned controls and a top-level operation strategy prescribing a desired set point for BLI fan shaft speed $\omega_{\text {mech }}^{*}$. Hence, no dedicated control of the turbofans is implemented.

\section{Results}

The scope of this paper is limited to the mechanisms of the proposed framework and motor modeling. Hence, no final statement and results regarding the actual performance of the application case are provided. However, the application case is used to qualitatively assess the effects of the previously introduced features on the transients to the steady state operating points. A similar approach is selected to compare the proposed motor efficiency modeling to a constant efficiency assumption.

Therefore, the electric machine efficiency is evaluated at the steady state operating points of the propulsion system obtained by the framework. Since these operating points strongly depend on the actual sizing of the components, the selected operation strategy and the operating point raster, the findings are strictly limited to this application case and a generalization cannot be obtained. As basis for the discussion, the maximum and the average absolute difference from the nominal efficiency of the generators and the motor are listed in Table $\mathbf{1 .}$

Table 1: Evaluation of the motor and generator efficiency for the application case and raster

\begin{tabular}{lcc}
\hline \hline Parameter & $\begin{array}{l}\text { Mean absolute difference from nominal } \\
\text { efficiency [\%] }\end{array}$ & $\begin{array}{l}\text { Maximum absolute difference from } \\
\text { nominal efficiency [\%] }\end{array}$ \\
\hline Motor & 0.61 & 1.03 \\
Generator & 0.29 & 0.66 \\
\hline \hline
\end{tabular}

As the maximum and average deviations from the nominal efficiency are below $1.1 \%$, the effect of a variable electric machine efficiency is negligible for the selected application case. Yet, transient operation, engine or fan start-up operating points are not represented in the considered operation range. Also, the turbofan deck only contains limited idle operation ranges. Hence, the deviation of the electric machine operating points from the design point is limited in means of minimal considered shaft speed and torque/current which partially explains the minor deviations from the nominal efficiency. Nonetheless, the approach allows to estimate the impact of the operating point dependent electric machine efficiency which is of minor importance for the captured operation range of the propulsion system performance deck in the application case. It is also interesting to mention, that the found mean absolute differences are comparable to the findings published in Ref. [1] omitting the low idle operation range.

Since the only control goal is to converge the system to a steady state operating point, a sophisticated control design can be avoided. This is especially true since the setpoints of the controllers are fixed over the simulated time and only a virtual system without any sensor noise or external distortion is governed. However, oscillations in the system should be avoided as they can increase the computational effort. Therefore, the pols should be placed well separated (similar to a cascaded control) by choosing the proportional and integral gains accordingly. Since the actual time constants of the system are not of interest for the proposed framework, the grid capacitance as well as the inertias of the shaft at the BLI fan and the low-pressure shaft can also be tuned together with the controller gains. As the motor and generator control are based on a constant grid voltage assumption, the voltage limits for the primary control $V_{l, \text { reduction }}$ and $V_{l, \text { cut-off }}$ are to be set close to the nominal value. Yet, they should allow for the controller to stay within the normal operation bounds in absence of a power insufficiency on the source side.

For the application case two exemplary convergence trajectories are displayed in Fig. 9. The plots are intended to reveal the qualitive properties of the proposed control and are used as qualitative representation of the transients. Hence, they are only displayed normalized by the respective steady state operation point and the approximate convergence time of the slowest transient in the secondary control.

In both cases, the three introduced states - low-pressure shaft speed, grid voltage and BLI-shaft speed - are displayed top to bottom. Both exemplary operating points represent ground operating cases. The left-hand case (subplots a, c, e Fig. 9), where the engine is set to the maximum fuel air ratio, is addressed first:

As depicted in subplot e of Fig. 9, the transient of the BLI shaft speed is set to be the slowest. The grid voltage state (subplot c Fig. 9) is initialized with the nominal voltage which is also the setpoint for the generator controls. Since no feed forward control is present, the generator control can only react to the current drawn by the motor after it manifests in a control error. At the beginning of the transient, the grid voltage drops consequently. As the generator is controlled to a faster dynamic than the motor, the PI-controllers governing the grid voltage restore the nominal voltage with a faster dynamic than the BLI-fan transient. As depicted in the upper subplot, the lowpressure shaft speed of the turbofan engines is set to have the fastest dynamic. It rises quickly from the initial value to a steady state operating point with negligible generator load. As the generators starts to fight the disturbance imposed on the grid by the motor, it increases the load on the low-pressure shaft and the turbofan adapts the steady state operating point to yield the related excess torque accordingly. 
The second case on the right-hand side (subplots b, d, f in Fig. 9 depicts the transients for a lower fuel air ratio. In this case, the pre-defined setpoint for the BLI shaft speed exceeds the power capability of the generators at the steady state low-pressure shaft speed of the turbofan engine. Due, to this mismatch the primary control has to take effect and sheds the BLI-fan load. Looking at the subplot b in Fig. 9, it can be deduced that turbofan again approaches a steady state operating point quickly. The generator, however, runs into the current limitation due to the slow shaft speed of the turbofan engine. Hence, the nominal grid voltage cannot be maintained (subplot $d$ in Fig. 9) and the voltage continuously drops while the BLI-shaft approaches its desired steady state operating point. After the grid voltage reaches the load reduction voltage $V_{l \text {,reduction }}$ (dashed red line) at about 5.5 times the convergence time of the BLI-shaft speed, the primary control takes effect and sheds the BLI-motor load by linearly reducing the power drawn by the BLI-motor with the decay in grid voltage until a balanced grid operation is found at about 10 times the convergence time of the BLI-shaft speed. At this point, a balanced steady state operating point is obtained (subplot b, d, f Fig. 9). Since the BLI-motor load is shed, the primary control also manifests in a transient of the BLI-shaft speed which concurrently drops to a steady state operating point below the desired shaft speed (subplot f Fig. 9).
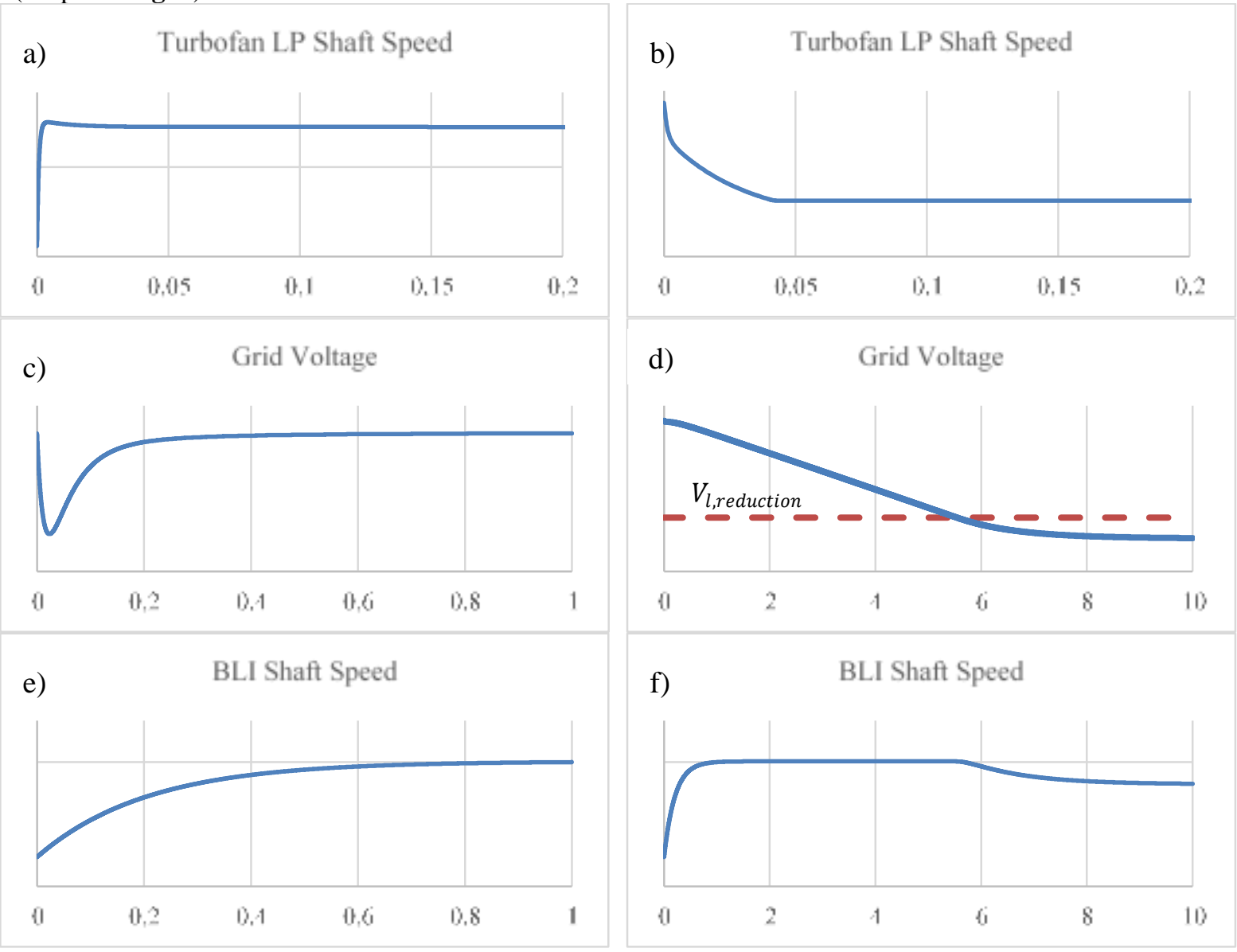

Fig. 9: Transients for the ground operation in case of the maximum fuel air ratio (a, c, e) and a low fuel air ratio $(b, d, f)$ for the states low-pressure shaft speed $(a, b)$, grid voltage $(c, d)$ and BLI shaft speed $(e, f)$ normalized by the steady state and the slowest transient

\section{Conclusion}

The paper proposes a simplified power law-based model for the electric machine which is build up on the basis of a standard equivalent circuit model. For the application case, the variation of the electric machine efficiency over the operation range is evaluated. The discrepancy between modeled motor/ generator efficiency and the nominal efficiency is assessed to be of minor importance for the application. However, this finding is limited to the selected application case.

The provided framework enables the generation of a performance deck for the desired hybrid electric aircraft configuration that spans its complete operation range. All relevant limitations affecting the allowable operation case are therein complied with. For the electric machines, compliance with the power and torque/current limitations are granted by the control scheme while the turbomachinery operation is restricted to the operation range of the input look-up tables via post-processing. Due to the simplistic management scheme, convergence to a steady state operation point is enabled even if the desired BLI-Fan operation exceeds the power capability of the power sources 
constituted by the generators mounted at the two turbofans. Hence, the approach allows for a rigorous definition of an operation strategy. Furthermore, it indicates that the approach does even find steady state operating points for mismatched component sizing. Due to these properties and the related plug and play capability, the approach is also adaptable to many other powertrain configurations.

The current approach uses the turbofan low-pressure shaft speed as a state and the fuel air ratio/ fuel flow to determine the excess torque of the turbofan. Yet, other engine control variables can influence the interrelation of these variables. This can lead to a non-bijective interrelation of low-pressure shaft speed and excess torque of the turbo fan at a given fuel air ratio/ fuel mass flow. If the shaft speed approaches an operation point with ambiguous excess torques, only one solution is considered and all others are disregarded. To avoid this, either the turbofan is controlled internally such that no ambiguity appears in the turbofan deck or the deck is set up with additional control variables such that a conclusive interrelation is achieved. For the sizing of the components in the considered test cases, no ambiguity was present with in the operation bounds of the propulsion system.

In future, the author envisions to improve the validity of the proposed motor efficiency modeling. Therefore, the range of applicability and the fidelity of the AC resistance model could be enhanced by following a more complex approach. Moreover, the windage loss estimation could be enhanced by looking more detailed at the actual integration and potentially accounting for forced air cooling. Also, splitting the stator core losses into hysteresis, eddy current and excess eddy current losses, or a modified Steinmetz equation as proposed in Ref. [18] should enhance the fidelity. Nonetheless, all these extensions require the predetermination of further parameters in the sizing tool and add complexity.

As mentioned before, the captured idle range is limited. Another study capturing this operation range can be conducted to ensure the applicability of the proposed control scheme. Adaptions of the turbofan deck generation are a powerful lever to surpass potential limitations of the approach as previously stated. Also, alternative implementations further detailing the dynamic behavior of the turbofan could be considered (e.g. Rowen's models).

A prosperous avenue for future work is to extend the current modeling environment with detailed converter models capturing the governing loss mechanisms and dynamics. Furthermore, the interest of the community in hydrogen-based technologies like fuel cell architectures is growing. This trend indicates further power train architectures to be investigated and component models to be integrated. As the architectures might feature dissimilar electric sources and multiple loads, a source side drop control in addition to the load side one can be implemented to increase the management capability of the approach and to implement a balancing for the dissimilar sources. Moreover, many shapes for the drop characteristic can be employed to introduce further management modes based on the grid voltage $[25,28]$ and pose interesting features for further source components like batteries [26].

The propulsion system models can be used as a starting point to create a full real-time simulation of the powertrain that is coupled with a flight dynamics model. However, proper solutions for the envelope protection of the turbo components need to be implemented as handling via post-processing is obviously inapplicable. The transient behavior of the system featuring the actual time constants of the used states can require more sophisticated control design.

\section{Acknowledgments}

The project leading to this application has received funding from the Clean Sky 2 Joint Undertaking under the European Union's Horizon 2020 research and innovation programme under grant agreement No CS2-GAM2016-2017-LPA.

The author would also like to thank the industry partner GE Aviation (Mohamed Osama, Andreas Peters) and Rolls-Royce (Andrew Smith, Clean Sky 2) for their interest in this topic and their guidance.

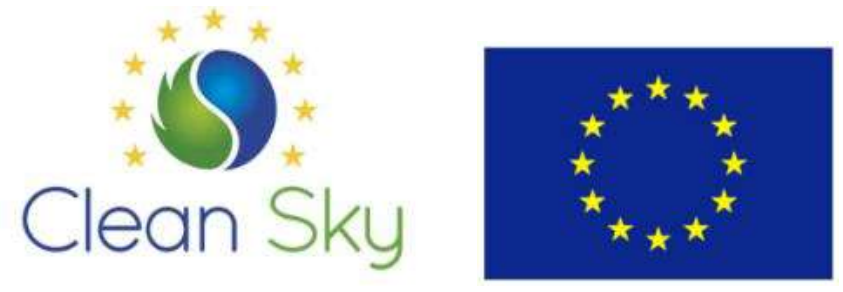

\section{References}

[1] P. Hanlon, G. L. Thomas, J. Csank und D. Sadey, „A Tool for Modeling and Analysis of Electrified Aircraft," in AIAA Propulsion and Energy 2019 Forum, Indianapolis, IN, 2019. 
[2] M. Iwanizki, M. J. Arzberger, M. Plohr, D. Silberhorn und T. Hecken, „Conceptual Design Studies of Short Range Aircraft Configurations with Hybrid Electric Propulsion,“ Dallas, Texas, 2019.

[3] D. Silberhorn, M. Arzberger, M. Mennicken, F. Wolters, C. Hollmann und M. Iwanizki, „Multidisciplinary Investigation of Partially Turboelectric, Boundary Layer Ingesting Aircraft Concepts, “ in AIAA Scitech 2020 Forum, Orlando, FL, 2020.

[4] F. Orefice, P. D. D. Vacchia und F. Nicolosi, „Aircraft Conceptual Design Including Powertrain System Architecture and Distributed Propulsion," in AIAA Propulsion and Energy 2019 Forum, Indianapolis, IN, 2019.

[5] A. Anderson, N. Renner, S. S. S. Agrawal, D. Lee, A. H. K. S. M. Banerjee und F. J.L., „System Weight Comparison of Electric Machine Topologies for Electric Aircraft Propulsion, " in 2018 AIAA/IEEE Electric Aircraft Technologies Symposium, Cincinnati, Ohio, 2018.

[6] X. Zhao, K. Kyprianidis, S. Sumsurooah, G. Valente, M. V. Rashed, C. Hill, C. Jacob, Gobbin, A., A. Bardenhagen, K. Prölss, M. Sielemann, J. Rantzer und E. Ekstedt, „A Framework for Optimization of Hybrid Aircraft,“ in Turbo Expo 2019: Turbomachinery Technical Conference and Exposition, Phoenix, Arizona, 2019.

[7] „CPACS standard,“ Deutsches Zentrum für Luft- und Raumfahrt e.V., [Online]. Available: https://cpacs.de/. [Zugriff am 07 2021].

[8] „European Commission, Flightpath 2050,“Europe’s Vision for Aviation, 2011.

[9] D. Silberhorn, C. Hollmann, M. Mennicken, F. Wolters, F. Eichner und M. Staggat, „Overall Design and Assessment of Aircraft concepts with Boundary Layer ingesting Engines, “ in German Aerospace Congress, Darmstadt, 2019.

[10] M. J. Arzberger und D. Zimmer, „A Modelica-based environment for the simulation of hybrid-electric propulsion systems, “ in Proceedings of the 13th International Modelica Conference, Regensburg, German, March 4-6, 2019.

[11] A. Mahmoudi, W. L. Soong, G. Pellegrino und E. Armando, „Efficiency maps of electrical machines,“ 2015 IEEE Energy Conversion Congress and Exposition (ECCE), pp. 2791-2799, 2015.

[12] G. Volpe, M. Popescu und F. G. J. Marignetti, „Modelling AC Winding Losses in a PMSM with High Frequency and Torque Density,“ 2018 IEEE Energy Conversion Congress and Exposition (ECCE), pp. 2300-2305, 2018.

[13] C. R. Sullivan, „Computationally efficient winding loss calculation with multiple windings, arbitrary waveforms, and two-dimensional or three-dimensional field geometry, "IEEE Transactions on Power Electronics, pp. 142-150, 2001.

[14] P. Mellor, R. Wrobel und N. Simpson, ,AC Losses in High Frequency Electrical Machine Windings formed from Large Section Conductors,“ 2014 IEEE Energy Conversion Congress and Exposition (ECCE), pp. 5563-5570, 2014.

[15] C. R. Sullivan, „Optimal choice for number of strands in a litz-wire transformer winding,“ IEEE Transactions on Power Electronics, pp. 283-291, 31999.

[16] A. Payne, „Skin Effect, Proximity Effect and the Resistance of Rectangular Conductors,“ 2016.

[17] A. Krings und J. Soulard, „Overview and Comparison of Iron Loss Models for Electrical Machines.," Journal of Electrical Engineering, pp. 162-169, 052010.

[18] J. Reinert, A. Brockmeyer und R. W. De Doncker, „Calculation of losses in ferro- and ferrimagnetic materials based on the modified Steinmetz equation," IEEE Transactions on Industry Applications, pp. 1055-1061, 2001.

[19] J. Lee, K. Nam, S. Choi und S. Kwon, „Loss Minimizing Control of PMSM with the Use of Polynomial Approximation,“ 2008 IEEE Industry Applications Society Annual Meeting, pp. 1-9, 2008.

[20] M. Popescu, D. G. Dorrell und D. M. Ionel, „A Study of the Engineering Calculations for Iron Losses in 3 phase AC Motor Models,“ IECON 2007 - 33rd Annual Conference of the IEEE Industrial Electronics Society, pp. 169-174, 2007.

[21] P. Dück und B. Ponick, „A novel iron-loss-model for permanent magnet synchronous machines in traction applications, “ 2016 International Conference on Electrical Systems for Aircraft, Railway, Ship Propulsion and Road Vehicles \& International Transportation Electrification Conference (ESARS-ITEC), pp. 1-6, 2016

[22] J. Huppunen, High-Speed Solid-Rotor Induction Machine - Electromagnetic Calculation and Design, Lappeenranta University of Technology, 2004.

[23] E. Bilgen und R. Boulos, „Functional Dependence of Torque Coefficient of Coaxial Cylinders on Gap Width and Reynolds Numbers,“ Journal of Fluids Engineering, pp. 122-126, Mar 1973. 
[24] F. Mink, N. Kubasiak, B. Ritter und A. Binder, „Parametric model and identification of PMSM considering the influence of magnetic saturation," 2012 13th International Conference on Optimization of Electrical and Electronic Equipment (OPTIM), pp. 444-452, 2012.

[25] Z. Shuai, J. Fang, F. Ning und Z. J. Shen, „Hierarchical structure and bus voltage control of DC microgrid,“ Renewable and Sustainable Energy Reviews, pp. 3670-3682, 2018.

[26] D. Chen und L. Xu, „Autonomous DC Voltage Control of a DC Microgrid With Multiple Slack Terminals,“ IEEE Transactions on Power Systems, pp. 897-1905, 2012.

[27] F. Gao, S. Bozhko, G. Asher, P. Wheeler und C. Patel, „An Improved Voltage Compensation Approach in a Droop-Controlled DC Power System for the More Electric Aircraft,“ IEEE Transactions on Power Electronics, pp. 7369-7383, Oct 2016.

[28] T. L. Vandoorn, B. Meersman, J. D. M. De Kooning und L. Vandevelde, „Analogy Between Conventional Grid Control and Islanded Microgrid Control Based on a Global DC-Link Voltage Droop, “ IEEE Transactions on Power Delivery, pp. 1405-1414, 2012.

[29] C. Ong, „Dynamic Simulation of Electric Machinery: Using MATLAB/SIMULINK,“ Prentice Hall PTR, 2003. 